\title{
Zur Theorie der differentiellen Thermokraft von Halbleitern
}

\author{
Von GüNter LaUtz
}

Aus dem Institut für technische Physik der Technischen Hochschule Braunschweig

(Z. Naturforschg. 8a, 361-371 [1953]; eingegangen am 10. April 1953)

In der allgemeinen Thermokraftformel des Zweibändermodells der Elektronentheorie bleibt als Parameter die Fermische Grenzenergie $\zeta$ unbestimmt. Diese ergibt sich erst aus der Ladungsträgerbilanz für den in Betracht kommenden Halbleiter. Selbst bei Beschränkung auf einen einfachen ,,klassischen“ Störstellenhalbleiter mit nur einem Störstellenniveau und nicht entartetem Elektronengas lassen sich die $\zeta$-Werte nur numerisch bestimmen. Jedoch kann man unter der Voraussetzung, daß der Bandabstand wenigstens dreimal so groß ist wie die Aktivierungsenergie der Störstellen, zwei allgemeine Formeln für $\zeta$ ableiten, die den gesamten Temperaturbereich lückenlos erfassen.

Die erste dieser Beziehungen gilt im Gebiet der reinen Störstellenhalbleitung, ohne daß sich eine Eigenleitung überlagert. Die mit diesem Ausdruck für die Fermische Grenzenergie aufgestellte Thermokraftformel umfaßt auch das Gebiet der vollständigen Ionisation und führt nur unter weiteren Näherungsannahmen, die eingehend diskutiert werden, zu den in der bisherigen Literatur vielfach benutzten Beziehungen für die Temperaturabhängigkeit der Thermokräfte.

Die zweite Näherungsformel für $\zeta$ gilt im Gebiet mittlerer und hoher Temperaturen. Sie erfaßt den Übergang von der Störstellen- zur Eigenhalbleitung einschließlich der Störstellenleitung bei vollständiger Ionisation und der Eigenhalbleitung und führt so auf eine Thermokraftformel mit einem entsprechend großen Gültigkeitsbereich. In der vorliegenden Arbeit wird ferner gezeigt, daß sich unter der bereits angegebenen Voraussetzung über die relative Größe der Aktivierungsenergie und des Bandabstandes die bei tiefen Temperaturen und die bei hohen Temperaturen gültigen Darstellungen für die Thermokraft in mittleren Temperaturen zu den gleichen Werten führen und sich so teilweise überdecken.

Die so ermittelten analytischen Formeln für die differentielle Thermokraft können zur Bestimmung der Aktivierungsenergie, des Bandabstandes oder der scheinbaren Massen der Ladungsträger benutzt werden, ohne daß die Experimente entweder im reinen Störleitungsgebiet oder im reinen Eigenleitungsgebiet ausgeführt zu werden brauchen. Bei der formalen Darstellung wird im Übergangsgebiet die Einführung eines temperaturabhängigen Verhältnisses der scheinbaren Massen von Elektronen und Defektelektronen nahegelegt. Die Gültigkeitsgrenzen einzelner Näherungsformeln werden an zwei Beispielen durch einen Vergleich mit exakten Lösungen veranschaulicht und im Zusammenhang mit dem gesamten Temperaturverlauf der Thermokraft erläutert.

Eine knappe Diskussion einzelner experimenteller Ergebnisse über die Temperaturabhängigkeit der 'Thermokraft zeigt eine qualitative Übereinstimmung mit den vorliegenden theoretischen Formeln. Doch reichen die experimentellen Angaben für einen quantitativen Vergleich in vielen Fällen nicht aus.

$Z^{n+1}$ ur Bestimmung der elektrischen Konstanten eines Halbleiters können Thermokraftmessungen einen wertvollen Beitrag liefern. So ermöglicht die Kombination derartiger Experimente mit dem HallEffekt die Ermittlung der scheinbaren Masse der Ladungsträger. Außerdem kann man aus der Temperaturabhängigkeit der Thermokraftwerte quantitative Aussagen über die Ladungsträgerkonzentrationen und die Aktivierungsenergien in Störstellenhalbleitern und eventuell über den Bandabstand eines Eigenhalbleiters erhalten. Dazu sind jedoch formelmäßige Zusammenhänge zwischen diesen Größen notwendig.

In der bisherigen Literatur werden vornehmlich zwei Formeln benutzt, von denen die eine für den
Störstellenhalbleiter und die andere für den Eigenhalbleiter gelten ${ }^{1}$. Doch werden diese Beziehungen häufig auch in den Fällen zur Berechnung der elektrischen Konstanten angewandt, in denen die Voraussetzungen für ihre Gültigkeit schon verletzt sind. Aus diesem Grunde werden wir in der vorliegenden Arbeit eine kritische Diskussion der einzelnen Näherungslösungen für die Temperaturabhängigkeit der Thermokraft vornehmen und zudem eine exakte Lösung mitteilen, die den gesamten Bereich von der reinen Störstellenhalbleitung bei nicht zu tiefen Temperaturen bis zur Eigenhalbleitung einschließlich darstellt. Damit können auch die Experimente

1 Z. B. A. H. Wilson, Metals and Semiconductors, Cambridge Univ. Press, London 1939. 
mit den theoretischen Formeln verglichen werden, die an Halbleitern gemacht werden, bei denen eine Überlagerung der Störstellen- und Eigenhalbleitung vorliegt. Das gilt z. B. beim Ge und Si sowie einigen halbleitenden intermetallischen Verbindungen infolge ihrer kleinen Bandabstände schon in den leicht zugänglichen Temperaturbereichen unter $1000^{\circ} \mathrm{K}$.

Dabei wollen wir uns in den nachfolgenden Ausführungen auf ,,klassische Halbleiter" beschränken. Die Ladungsträgerkonzentrationen sollen somit stets kleiner als die kritische Trägerdichte $n^{0}$ bleiben, so daß keine Entartung im Elektronengas auftritt. Diese Voraussetzung ermöglicht eine erhebliche Verringerung des Rechenaufwandes, da die Fermische Verteilungsfunktion in den Leitungsbändern durch eine Boltzmannsche e-Funktion ersetzt werden kann. Zur weiteren Vereinfachung wollen wir die Berechnungen für das Modell eines reinen Überschußhalbleiters ausführen, bei dem das Donatorenniveau im Energiebändermodell wenigstens $4 k T$ ( $k=$ Boltzmannsche Konstante, $T=$ absoluteTemperatur) unter dem unteren Rand des Leitungsbandes liegt. Der Sonderfall, in dem das Elektronenspenderniveau zwischen das Leitungsband und die angegebene Grenze fällt, ist in bezug auf die Temperaturabhängigkeit der Thermokraft in einer Arbeit von $\mathrm{Y}$ amashita ${ }^{2}$ näher diskutiert worden, so daß hier auf diese Literatur verwiesen werden kann. Die angegebenen Beschränkungen sind nicht wesentlich und bei vielen Halbleiterstoffen erfüllt.

Um den Einfluß der Eigenhalbleitung mitzuerfassen, werden wir von den allgemeinen Formeln des Zweibändermodells der Elektronentheorie ausgehen. Diese enthalten als einen noch zu bestimmenden Parameter die Fermische Grenzenergie ל, deren Wert aus der Ladungsträgerbilanz meist nur durch numerische Rechnungen für bestimmte Beispiele zu ermitteln ist. Der einzuschlagende Rechengang wird daher von den allgemeinen Formeln des Zweibändermodells ausgehend über die Bestimmung des $\zeta$ Wertes zu einer expliziten Darstellung der Temperaturabhängigkeit der absoluten Thermokraft führen.

1. Die allgemeinen Formeln für die absolute Thermokraft im Zweibändermodell der Elektronentheorie

In einer Halbleiterprobe, deren Dimensionen groß gegenüber der freien Weglänge der Elektronen bleiben sollen, möge ein elektrisches Feld und ein Temperaturgefälle in der $x$-Richtung vorhanden sein.
Dann gilt für die Verteilungsfunktion der Ladungsträger im stationären Fall die Boltzmannsche Fundamentalgleichung in der Form

$$
v_{x} \frac{\partial f}{\partial x}+b_{x} \frac{\partial f}{\partial v_{x}}=\left(\frac{\partial f}{\partial t}\right)_{\text {Stöße }} .
$$

Dabei sind $v_{x}$ und $b_{x}$ die Geschwindigkeit resp. Beschleunigung der Ladungsträger und $f$ die gestörte Verteilungsfunktion. Läßt sich die Wechselwirkung der Elektronen und die der Defektelektronen mit dem Gitter durch zwei einheitliche Relaxationszeiten $\tau_{\mathrm{e}}$ und $\tau_{\mathrm{h}}$ beschreiben, so können wir den Lorentzschen Ansatz

$$
\left(\frac{\partial f}{\partial t}\right)_{\text {Stöße }}=-\frac{f-f_{0}}{\tau}
$$

einführen, wobei $\tau$ durch $\tau_{\mathrm{e}}$ oder $\tau_{\mathrm{h}}$ zu ersetzen ist, je nachdem sich die Rechnungen auf Elektronen oder Defektelektronen beziehen. $f_{0}$ stellt die ungestörte Verteilungsfunktion dar.

In der üblichen Näherung ersetzt man auf der linken Seite der Boltzmann-Gleichung $f$ durch $f_{0}$ und erhält mit

$$
\begin{gathered}
f_{1}=f-f_{0}, f_{1} \ll f_{0}, \\
v_{x} \frac{\partial f_{0}^{\mathrm{e}}}{\partial x}-\frac{e F_{x} x}{m_{\mathrm{e}}{ }^{*}} \frac{\partial f_{0}^{\mathrm{e}}}{\partial v_{x}}=-\frac{f_{1}^{\mathrm{e}}}{\tau_{\mathrm{e}}} .
\end{gathered}
$$

Eine entsprechende Differentialgleichung läßt sich für die Defektelektronen im Valenzband angeben:

$$
v_{x} \frac{\partial f_{0}^{\mathrm{h}}}{\partial x}+\frac{e F_{x}}{\left|m_{\mathrm{h}}{ }^{*}\right|} \frac{\partial f_{0}^{\mathrm{h}}}{\partial v_{x}}=-\frac{f_{1}^{\mathrm{h}}}{\tau_{\mathrm{h}}} .
$$

Dabei bedeuten $m_{\mathrm{e}}{ }^{*}$ und $\left|m_{\mathrm{h}}{ }^{*}\right|$ die positiven scheinbaren Massen der Elektronen bzw. Defektelektronen, $e$ die elektrische Elementarladung und $F_{x}$ die elektrische Feldstärke in der $x$-Richtung. Die für die Elektronen und Defektelektronen unterschiedlichen Verteilungsfunktionen sind durch einen oberen Index gekennzeichnet.

Unter der Voraussetzung einer isotropen Verteilung bekommen wir nach bekannten Umrechnungen und nach Einführung der Energie $E$ der Ladungsträger mit

$$
F_{x}^{*}=F_{x}+\frac{1}{e} \frac{\partial \zeta}{\partial x}
$$

als Lösung der Differentialgl. (1 a)

$$
f_{1}^{\mathrm{e}}=\left(e v_{x} \tau_{\mathrm{e}} F_{x}^{*}+v_{x} \tau_{\mathrm{e}} \frac{E-\zeta}{T} \frac{\partial T}{\partial x}\right) \frac{\partial f_{0}^{\mathrm{e}}}{\partial E}
$$

2 J. Yamashita, Jap. J. Physics. 4, 310 [1949]. Leider war dem Autor diese Arbeit nur auszugsweise zugänglich. 
und eine entsprechende Gleichung mit den oberen Indizes $\mathrm{h}$ statt $\mathrm{e}$. Als weitere Bedingung für die Berechnung der Thermokraft hat man aus physikalischen Gründen zu fordern, daß der Gesamtstrom und damit auch die über den Querschnitt gemittelte Stromdichte verschwinden. Da die gestörten Verteilungsfunktionen von den Ortskoordinaten nicht abhängen, wird der Mittelwert der Stromdichte gleich der Stromdichte selbst. Unter Berücksichtigung der Elektrizitätsleitung in zwei Bändern gilt:

$$
\begin{aligned}
j_{x}=0=-e \iint v_{x} f_{1}{ }^{\mathrm{e}} D^{\mathrm{e}}(E) \mathrm{d} E \mathrm{~d} \Omega \\
+e \iint v_{x} f_{1}{ }^{\mathrm{h}} D^{\mathrm{h}}(E) \mathrm{d} E \mathrm{~d} \Omega,
\end{aligned}
$$

wobei wie üblich

$$
\begin{aligned}
& D^{\mathrm{e}}(E)=\left(\frac{2 m_{\mathrm{e}}{ }^{*}}{h^{2}}\right)^{3 / 2}\left(E-E_{2}\right)^{1 / 2} \quad \text { mit } E>E_{2}, \\
& D^{\mathrm{h}}(E)=\left(\frac{2\left|m_{\mathrm{h}}{ }^{*}\right|}{h^{2}}\right)^{3 / 2}\left(E_{1}-E\right)^{1 / 2} \quad \text { mit } E<E_{1}
\end{aligned}
$$

die Zustandsdichten in der Raumwinkeleinheit am unteren Rande $E_{2}$ des Leitfähigkeitsbandes bzw. am oberen Rande $E_{1}$ des Valenzbandes bedeuten.
Nach den in der Einleitung angegebenen Beschränkungen auf ,,klassische Halbleiter" können wir die Verteilungsfunktionen im ungestörten $\mathrm{Zu}$ stand bis auf einen Fehler $<1 \%$ durch einfache $e$ Funktionen ersetzen:

$f_{0}{ }^{\mathrm{e}}=\frac{1}{1+e^{\frac{E-\zeta}{k T}}}=e^{-\frac{E-\zeta}{k T}}$, wenn $\frac{E-\zeta}{k T}>4$
$f_{0}{ }^{\mathrm{h}}=\frac{e^{\frac{E-\zeta}{k T}}}{1+e^{\frac{E-\zeta}{k T}}}=e^{\frac{E-\zeta}{k T}}$, wenn $\frac{E-\zeta}{k T}<-4$. Unter diesen Voraussetzungen muß die Fermische Grenzenergie $\zeta$ also wenigstens $4 k T$ vom unteren Rand des Leitfähigkeitsbandes und $4 k T$ vom oberen Rand des Valenzbandes entfernt sein. Andernfalls ist die Einführung der Boltzmannschen Verteilungsfunktion nicht zulässig.

Setzt man nun unter Berücksichtigung von (7) die gestörten Verteilungsfunktionen (3) in (4) ein, so ergibt sich für die thermoelektrische Feldstärke nach bekannten Umformungen ${ }^{3}$ :

$F_{x}{ }^{*}=-\frac{1}{e T} \frac{\partial T}{\partial x} \frac{\left\{\left(m_{\mathrm{e}}{ }^{*}\right)^{1 / 2} e^{-\frac{E_{2}-\zeta}{k T}}\left[I_{5 / 2}^{\mathrm{e}}-\left(\zeta-E_{2}\right) I_{3 / 2}^{\mathrm{e}}\right]-\left|m_{\mathrm{h}}{ }^{*}\right|^{1 / 2} e^{\frac{E_{1}-\zeta}{k T}}\left[I_{5 / 2}^{\mathrm{h}}-\left(E_{1}-\zeta\right) I_{3 / 2}^{\mathrm{h}}\right]\right\}}{\left(m_{\mathrm{e}}{ }^{*}\right)^{1 / 2} e^{-\frac{E_{2}-\zeta}{k T}} I_{3 / 2}^{\mathrm{e}}+\left|m_{\mathrm{h}}{ }^{*}\right|^{1 / 2} e^{\frac{E_{1}-\zeta}{k T}} I_{3 / 2}^{\mathrm{h}}}$.

Als Abkürzung wurden dabei nachstehende Beziehungen eingeführt:

$$
I_{n / 2}^{\mathrm{e}}=\int_{E_{2}}^{\infty} \tau_{\mathrm{e}}(E)\left(E-E_{2}\right)^{n / 2} e^{-\frac{E-E_{2}}{k T}} \mathrm{~d} E, I_{n / 2}^{\mathrm{h}}=\int_{-\infty}^{E_{1}} \tau_{\mathrm{h}}(E)\left(E_{1}-E\right)^{n / 2} e^{-\frac{E_{1}-E}{k T}} \mathrm{~d} E .
$$

Diese Integrale lassen sich erst nach besonderen Annahmen über die Stoßzeiten $\tau_{\mathrm{e}}$ und $\tau_{\mathrm{h}}$ weiter auswerten.

Nun wird in der Elektronentheorie gezeigt, daß der Faktor von $\partial T / \partial x$ in der Beziehung (7) gleich der absoluten Thermokraft $\varepsilon$ ist. Damit folgt als allgemeiner Ausdruck für $\varepsilon$ im Zweibändermodell bei einem ,,klassischen Halbleiter“:

$\varepsilon=-\frac{1}{e T} \frac{\left\{\left(m_{\mathrm{e}}{ }^{*}\right)^{1 / 2} e^{-\frac{E_{2}-\zeta}{k T}}\left[I_{5 / 2}^{\mathrm{e}}-\left(\zeta-E_{2}\right) I_{3 / 2}^{\mathrm{e}}\right]-\left|m_{\mathrm{h}}{ }^{*}\right|^{1 / 2} e^{\frac{E_{1}-\zeta}{k T}}\left[I_{5 / 2}^{\mathrm{h}}-\left(E_{1}-\zeta\right) I_{3 / 2}^{\mathrm{h}}\right]\right\}}{\left(m_{\mathrm{e}}{ }^{*}\right)^{1 / 2} e^{-\frac{E_{2}-\zeta}{k T}} I_{3 / 2}^{\mathrm{e}}+\left|m_{\mathrm{h}}{ }^{*}\right|^{1 / 2} e^{\frac{E_{1}-\zeta}{k T}} I_{3 / 2}^{\mathrm{h}}}$.

Von dieser allgemeinen Formel (9) kommt man sehr schnell zur einfacheren Gleichung für den reinen Überschußhalbleiter, da in diesem Spezialfall $\left|E_{1}-\zeta\right| \gg\left|E_{2}-\zeta\right|$ wird. Man erhält:

$$
\varepsilon=-\frac{1}{e T}\left[\frac{I_{5 / 2}^{\mathrm{e}}}{I_{3 / 2}^{\mathrm{e}}}-\left(\zeta-E_{2}\right)\right]
$$

und bei einem Mangelhalbleiter

$$
\varepsilon=\frac{1}{e T}\left[\frac{I_{5 / 2}^{\mathrm{h}}}{I_{3 / 2}^{\mathrm{h}}}-\left(E_{1}-\zeta\right)\right] .
$$

Zur weiteren Auswertung des Zusammenhanges zwischen $\varepsilon$ und den Halbleiterkonstanten und der Temperatur $T$ benötigt man eine explizite Darstellung der Fermischen Grenzenergie $\zeta$.

${ }^{3}$ Z. B. H. Fröhlich, Elektronentheorie der Metalle, Verlag Springer, Berlin 1936. 
2. Die Berechnung der Fermischen Grenzenergie

Als Ausgangsgleichung dient uns die Ladungsträgerbilanz, die für einen Halbleiter mit nur einem Donatorniveau

$$
n_{\mathrm{e}}=n_{\mathrm{h}}+\frac{n_{\mathrm{1}}}{1+e^{-\frac{E_{2}^{\prime}-\xi}{k T}}}
$$

und für einen Halbleiter mit nur einem Acceptorniveau

$$
n_{\mathrm{e}}=n_{\mathrm{h}}-\frac{n_{\mathrm{A}}}{1+e^{\frac{E_{1}^{\prime}-5}{k T}}}
$$

lautet. Dabei bedeuten $n_{\mathrm{e}}$ die Elektronenkonzentration im Leitungsband und $n_{\mathrm{h}}$ die der Defektelektronen im Valenzband. $n_{\mathrm{D}}$ und $n_{\mathrm{A}}$ sind die Donator- bzw. Acceptorkonzentrationen und $\mathrm{E}_{2}{ }_{2}$ bzw. $E^{\prime}{ }_{1}$ die diesen entsprechenden Energiewerte (Abb.1).

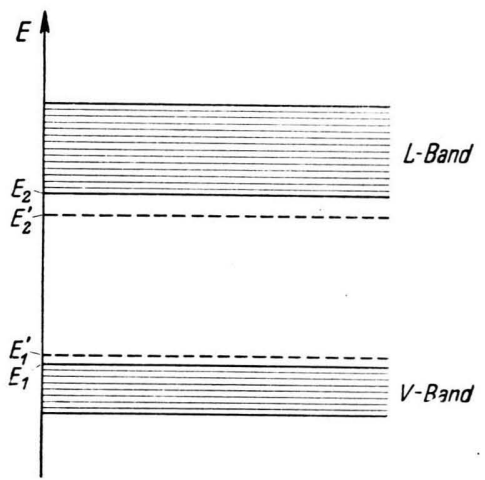

Abb. 1. Energieschema eines Halbleiters mit Donatorund Acceptortermen.

Nach den bereits angegebenen Voraussetzungen über die Lage der Fermi-Energie $\zeta$ gilt bekanntlich ${ }^{3}$ :

$n_{\mathrm{e}}=n_{-}{ }^{0} e^{-\frac{\boldsymbol{E}_{2}-\xi}{k T}}$ mit $n_{-}{ }^{0}=2\left(\frac{2 \pi m_{\mathrm{e}}{ }^{*} k T}{h^{2}}\right)^{3 / 2}$

und

$n_{\mathrm{h}}=n_{+}{ }^{0} e^{\frac{E_{1}-\xi}{k T}} \operatorname{mit} n_{+}{ }^{0}=2\left(\frac{2 \pi\left|m_{\mathrm{h}}{ }^{*}\right| k T}{h^{2}}\right)^{3 / 2}$.

Da die einfachen Beziehungen (10a) und (10b) bereits kubische Gleichungen in $\exp (\zeta / k T)$ darstellen, ist eine im ganzen Temperaturbereich gültige allgemeine Lösung für $\zeta$ nur numerisch möglich. In vielen praktischen Fällen lassen sich die Gln. (10a) und (10 b) jedoch so weit vereinfachen, daß quadratische Beziehungen in $\exp (\zeta / k T)$ entstehen. Dabei wollen wir im folgenden zwei Näherungslösungen diskutieren, die besondere Bedeutung haben. So betrachten wir zunächst den Spezialfall eines idealen Störstellenhalbleiters, bei dem die Eigenleitung nicht merkbar ist. Diese Voraussetzung trifft vornehmlich bei den Halbleitern zu, bei denen die Aktivierungsenergie $\Delta E_{\mathrm{D}}=E_{2}-E^{\prime}{ }_{2} \ll \Delta B=E_{2}-E_{1}$ bleibt. Je größer $A B$ ist, zu um so höheren Temperaturen reicht die Gültigkeit der benutzten Näherung. Die so abgeleiteten Formeln werden bei allen einfachen Störstellenhalbleitern im Gebiet tiefer Temperaturen gültig sein.

Überlagert sich bei einer Temperatursteigerung schließlich die Eigenhalbleitung, so läßt sich eine zweite Näherungsformel finden, die $\zeta$ im Gebiet hoher Temperaturen exakt darzustellen vermag. Damit diese beiden Beziehungen für $\zeta$ lückenlos aneinander passen oder sich zum Teil überdecken, muß $\Delta E_{\mathrm{D}} \leq 2 / 3 \cdot \Delta B / 2$ bleiben, wie wir weiter unten zeigen werden. Diese Voraussetzung ist aber bei sehr vielen Halbleitern erfüllt, so daß in dieser Weise eine Darstellung der Temperaturabhängigkeit der Fermischen Grenzenergie $\zeta$ ohne eine allgemeine Lösung einer der kubischen Gln. (10a) oder (10 b) möglich ist.

Beschränken wir uns zunächst auf den idealen Überschußhalbleiter, für den $\Delta E_{\mathrm{D}} \ll \Delta B$ ist, so gilt statt (10a):

$$
n_{-}{ }^{0} e^{-\frac{E_{2}-\xi}{k T}}=\frac{n_{\mathrm{D}}}{1+e^{-\frac{E_{2}^{\prime}-\xi}{k T}}} .
$$

Daraus erhält man für $\zeta$ durch einfache Rechnung:

$$
\zeta=E_{2}{ }^{\prime}+k T \ln \left\{\frac{1}{2} \sqrt{1+4 \frac{n_{\mathrm{p}}}{n_{-}{ }^{0}} e^{\frac{k E_{\mathrm{D}}}{k T}}}-\frac{1}{2}\right\} .
$$

Bei der praktischen Auswertung der Temperaturabhängigkeit der Thermokraft interessieren zwei Näherungsformeln, die sich aus der Gl. (13) ableiten lassen. So gilt z. B. für kleine $T$ :

$$
\left|\frac{n_{\mathrm{D}}}{n_{-}{ }^{0}} e^{\frac{\Delta E_{\mathrm{D}}}{k T}}\right| \gg 1 \text {. }
$$

Unter Beachtung von

$$
\ln (x-1)=\ln x-\frac{1}{x} \text { für } x \gg 1
$$

folgt dann aus (13):

$$
\begin{gathered}
\zeta=\frac{E_{2}+E_{2}{ }^{\prime}}{2}+\frac{k T}{2} \ln \frac{n_{\mathrm{D}}}{n_{-}{ }^{0}}-\frac{k T}{2}\left(\frac{n_{-}{ }^{0}}{n_{\mathrm{D}}}\right)^{1 / 2} \\
\cdot e^{-\frac{k E_{\mathrm{D}}}{2 k T}} .
\end{gathered}
$$

Das ist bis auf den letzten Summanden, der nur eine kleine Korrektur darstellt, die für Störstellen- 
halbleiter bekannte Beziehung ${ }^{3}$. Außerdem wird im Gebiet höherer Temperaturen:

$$
\left|\frac{n_{\mathrm{D}}}{n_{-}{ }^{0}} e^{\frac{\Delta E_{\mathrm{D}}}{k T}}\right| \ll 1
$$

und damit

$\zeta=E_{2}+k T \ln \frac{n_{\mathrm{D}}}{n_{-}{ }^{0}}-k T \frac{n_{\mathrm{p}}}{n_{-}{ }^{0}} e^{\frac{1 E_{\mathrm{D}}}{k T}}$,

wobei wiederum der letzte Summand eine kleine Korrektur darstellt. Da in (13b) die Aktivierungsenergie $\Delta E_{\mathrm{D}}$ oder der Energiewert $E^{\prime}{ }_{2}$ des Störstellentermes höchstens noch in dem Korrektionsglied auftreten, gilt diese Formel im Gebiet vollständiger Ionisation aller Störstellen.

Wollen wir den Gültigkeitsbereich einer der Gln. (13a) oder (13b) durch die Lage des $\zeta$-Wertes charakterisieren, so sieht man leicht, daß (13a) dann zur Berechnung von $\zeta$ mit einem Fehler $<1 \%$ anwendbar ist, wenn $\zeta$ wenigstens $4 k T$ über dem Störstellenniveau $E^{\prime}{ }_{2}$ liegt. Außerdem muß nach den Voraussetzungen, die zur Aufstellung der Gl. (10a) führten, noch gefordert werden, daß $\zeta$ auch wenigstens $4 k T$ unter dem unteren Rand des Leitungsbandes bleibt. Das ist von Hutner, Rittner und Du Pré eingehend diskutiert worden ${ }^{4}$. Gl. (13b) hingegen können wir dann mit der nämlichen Genauigkeit zur Berechnung von $\zeta$ benutzen, wenn $\zeta$ wenigstens $4 k T$ unter dem Donatorenniveau $E^{\prime}{ }_{2}$ bleibt.

Die Größe der Temperaturintervalle, in denen eine der Näherungsgl. (13a) oder (13b) bei einem reinen Überschußhalbleiter gültig ist, hängt in sehr starkem Maße von der Lage des Störstellenniveaus und von der Störstellenkonzentration ab. $\mathrm{Zu}$ bemerken bleibt noch, daß $\zeta$ bei reiner Störstellenhalbleitung aus (11a) auch in der Form

$$
\zeta=E_{2}+k T \ln \frac{n_{\mathrm{e}}}{n_{-}{ }^{0}}
$$

ausgedrückt werden kann. Doch ist in dieser Beziehung allgemein $n_{\mathrm{e}}$ durch

$$
\begin{aligned}
n_{\mathrm{e}} & =n_{-}{ }^{0} e^{-\frac{E_{2}-\zeta}{k T}} \\
& =n_{-}{ }^{0} e^{-\frac{\Delta E_{\mathrm{D}}}{k T}}\left(\frac{1}{2} \sqrt{1+4 \frac{n_{\mathrm{D}}}{n_{-}{ }^{0}} e^{\frac{\Delta E_{\mathrm{D}}}{k T}}}-\frac{1}{2}\right)
\end{aligned}
$$

zu ersetzen. Nur in dem Gültigkeitsbereich der Gl. (13a) können wir in der üblichen Weise

setzen.

$$
n_{\mathrm{e}}=\left(n_{-}^{0} n_{\mathrm{D}}\right)^{1 / 2} e^{-\frac{\Delta E_{\mathrm{D}}}{2 k T}}
$$

${ }^{4}$ R. A. Hutner, E. S. Rittner u. F. K. Du Pré, Philips Res. Rep. 5, 188 [1950].
Beachten wir, daß nach (13) $\zeta$ mit zunehmender Temperatur abnimmt, so werden wir schließlich bei Temperaturerhöhungen in ein Gebiet kommen, wo die Eigenleitung sich der Störstellenleitung mehr und mehr überlagert. Um zu einer quadratischen Gleichung zur Bestimmung von $\exp (\zeta / k T)$ zu kommen, wollen wir fordern, daß dieser Übergang erst in einem Temperaturintervall stattfindet, in dem die Störstellen bereits vollständig ionisiert sind. Dann schließt sich die im folgenden abgeleitete Beziehung für $\zeta$ lückenlos an die durch Gl. (13) dargestellte Abhängigkeit an, wobei sich beide Funktionen in mittleren Temperaturen vollständig überdecken. Die so eingeführte Annahme enthält implizit eine Aussage über das Verhältnis der Aktivierungsenergie $\triangle E_{\mathrm{D}}$ zur Größe des Bandabstandes $\triangle B$. Das werden wir im Anschluß an die allgemeinen Ableitungen quantitativ formulieren. Im Übergangsgebiet gilt also:

oder

$$
n_{\mathrm{e}}=n_{\mathrm{D}}+n_{\mathrm{h}}
$$

$$
e^{-\frac{E_{2}-\xi}{k T}}=\frac{n_{\mathrm{p}}}{n_{-}{ }^{0}}\left(1+\frac{n_{+}{ }^{0}}{n_{\mathrm{D}}} e^{\frac{E_{1}-\zeta}{k T}}\right) .
$$

Daraus folgt:

$$
\begin{aligned}
\zeta & =E_{2}+k T \ln \frac{n_{\mathrm{D}}}{n_{-}{ }^{0}} \\
& +k T \ln \left\{\frac{1}{2}+\frac{1}{2} \sqrt{\left.1+4 \frac{n_{+}{ }^{0} n_{-}{ }^{0}}{n_{\mathrm{D}}{ }^{2}} e^{-\frac{A B}{k T}}\right\} .}\right.
\end{aligned}
$$

Mit Hilfe dieser Beziehung gelingt es so, den ganzen Übergang von der Störstellen- zur Eigenhalbleitung mit Einschluß der Störstellenleitung bei vollständiger Ionisation und der Eigenleitung durch eine einzige Formel zu erfassen. Auch hier interessieren zwei Näherungen. Einmal kann beim Beginn des Überganges die Eigenleitung als geringe Störung der Störstellenleitung aufgefaßt werden. Dann ist:

$$
\left|\frac{n_{+}{ }^{0} n_{-}{ }^{0}}{n_{\mathrm{D}}{ }^{2}} e^{-\frac{A B}{k T}}\right| \ll 1
$$

und

$\zeta=E_{2}+k T \ln \frac{n_{\mathrm{D}}}{n_{-}{ }^{0}}+k T \frac{n_{+}{ }^{0} n_{-}{ }^{0}}{n_{\mathrm{D}}{ }^{2}} e^{-\frac{1 B}{k T}}$.

Zum anderen aber wird im Eigenleitungsgebiet sich der Anteil der aus dem Donatorniveau stammenden Elektronen als kleine Störung bemerkbar machen. Hier gilt:

$$
\left|\frac{n_{+}{ }^{0} n_{-}{ }^{0}}{n_{\mathrm{D}}{ }^{2}} e^{-\frac{\Delta B}{k T}}\right| \gg 1
$$

und damit 


$$
\begin{aligned}
\zeta=\frac{E_{1}+E_{2}}{2} & +\frac{3}{4} k T \ln \left(\frac{\left|m_{\mathrm{h}}{ }^{*}\right|}{m_{\mathrm{e}}{ }^{*}}\right) \\
& +\frac{k T}{2} \frac{n_{\mathrm{p}}}{\left(n_{+}{ }^{0} n_{-}{ }^{0}\right)^{1 / 2}} \cdot e^{\frac{\Delta B}{2 k T}} .
\end{aligned}
$$

Bis auf das letzte Glied ist dieser Ausdruck mit dem $\zeta$-Wert für die reine Eigenleitung identisch. Wie groß der Einfluß des Korrektionsgliedes auf die Fermische Grenzenergie sein kann, ist aus dem folgenden Zahlenbeispiel ersichtlich: Bei 0,5 eV Bandabstand und einer Störstellenkonzentration von $5 \cdot 10^{15}$ $\mathrm{cm}^{-3}$ wird der letzte Summand bereits größenordnungsmäßig gleich $k T$. Das entspricht bei $550^{\circ} \mathrm{K}$ schon einem Wert von 0,05 eV, d. h. 10\% des Bandabstandes.

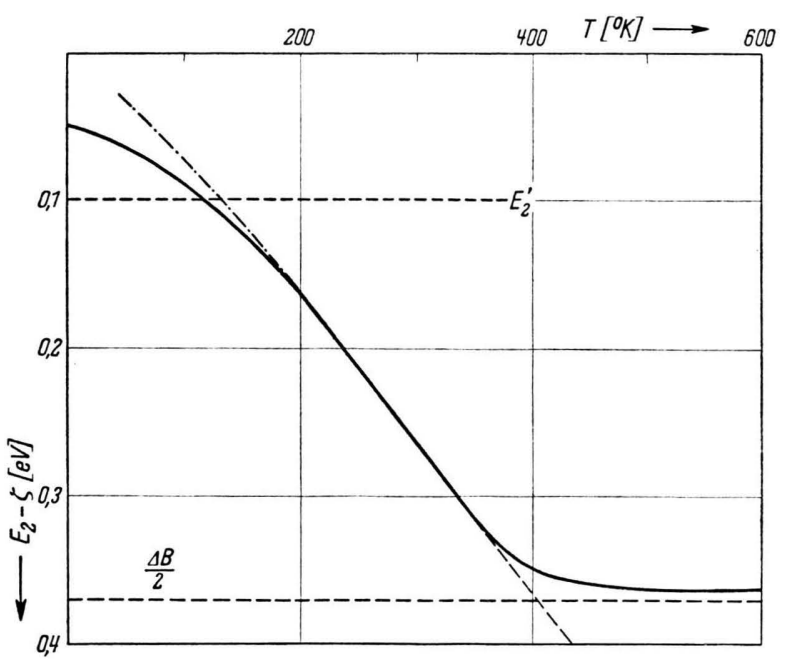

Abb. 2. Temperaturabhängigkeit der Fermischen Grenzenergie für einen einfachen Überschußhalbleiter. $\left|m_{\mathrm{h}}^{*}\right| / m_{\mathrm{e}}^{*}=1,2 ; n_{\mathrm{D}}=10^{15} \mathrm{~cm}^{-3} ; \Delta B=0,74 \mathrm{eV} ; \Delta E_{\mathrm{D}}$ $=0,1 \mathrm{eV}$. - - - nach Gl. (13), - $-\cdot-$ nach Gl. (19), numerisch nach (10 a) berechnete Werte.

In Abb. 2 ist die Temperaturabhängigkeit der Fermischen Grenzenergie für Ge dargestellt, bei dem ein Bandabstand von 0,74 eV und ein Donatorniveau $0,1 \mathrm{eV}$ unter dem Rand des Leitfähigkeitsbandes angenommen sind. Die Störstellenkonzentration betrage $10^{15} \mathrm{~cm}^{-3}$. Das Verhältnis der scheinbaren Massen möge

$$
\frac{\left|m_{\mathrm{h}}{ }^{*}\right|}{m_{\mathrm{e}}{ }^{*}}=1,2
$$

sein. Die Voraussetzung einer lückenlosen Aneinanderschließung der Darstellungen (13) und (19) sind mit $\triangle E_{\mathrm{D}}<0,24 \mathrm{eV}$ noch erfüllt. Die einzelnen $\zeta$-Werte sind im Störleitungsgebiet nach Gl. (13) bestimmt worden. Von $200^{\circ} \mathrm{K}$ an aufwärts stimmen alle $\zeta$-Werte bereits mit den nach Gl. (19) berechneten überein.

Nunmehr können wir auch die Voraussetzung quantitativ angeben, die bezüglich der Größe von $\Delta E_{\mathrm{D}}$ und $\Delta B$ erfüllt sein muß, damit die oben abgeleiteten Formeln für eine lückenlose Überdeckung des gesamten Temperaturbereiches ausreichen. Dann müssen die nach Gl. (13b) bestimmten $\zeta$ Werte mit den nach (19a) berechneten im Gebiet mittlerer Temperaturen übereinstimmen. Also muß gelten:

$$
\left|\frac{n_{-}{ }^{0}}{n_{\mathrm{D}}} e^{-\frac{\Delta E_{\mathrm{D}}}{k T}}\right| \gg 1 \gg\left|\frac{n_{+}{ }^{0} n_{-}{ }^{0}}{n_{\mathrm{1}}{ }^{2}} e^{-\frac{\Delta B}{k T}}\right| .
$$

Da die erste Teilbeziehung sicher erfüllt ist, wenn die linke Seite der Ungleichung $\geq 10$ und die rechte Seite $\leq 1 / 10$ ist, kommen wir zu der Abschätzung

$$
2 \frac{\Delta E_{\mathrm{D}}}{k T} \leq \frac{\Delta B}{k T}+\ln \frac{n_{\mathrm{D}}}{n_{-}{ }^{0}},
$$

wobei im allgemeinen $n_{+}{ }^{0} \leq n_{-}{ }^{0}$ gelten wird. Ferner muß

$$
\ln \frac{n_{\mathrm{D}}}{n_{-}{ }^{0}}+\frac{\Delta E_{\mathrm{D}}}{k T} \leq 0
$$

sein. Also folgt die bereits erwähnte Ungleichung:

$$
\frac{2}{3} \frac{\Delta B}{2} \geq \Delta E_{\mathrm{D}}
$$

als Voraussetzung für ein lückenloses Aneinanderschließen der Gln. (13) und (19) zur Berechnung von $\zeta$.

Zum Abschluß dieses Kapitels wollen wir noch die Ladungsträgerkonzentrationen in den beiden Bändern berechnen, wenn $\zeta$ durch den Ausdruck (19) gegeben ist. Aus (11 a) und (11 b) ergibt sich:

$$
\begin{aligned}
& n_{\mathrm{e}}=n_{\mathrm{D}}\left(\frac{1}{2}+\frac{1}{2} \sqrt{1+\frac{n_{+}{ }^{0} n_{-}{ }^{0}}{n_{\mathrm{D}}{ }^{2}} e^{-\frac{A B}{k T}}}\right), \\
& n_{\mathrm{h}}=\frac{\frac{n_{+}{ }^{0} n_{-}{ }^{0}}{n_{\mathrm{D}}} e^{-\frac{1 B}{k T}}}{\frac{1}{2}+\frac{1}{2} \sqrt{1+\frac{n_{+}{ }^{0} n_{-}{ }^{0}}{n_{\mathrm{D}}{ }^{2}} e^{-\frac{1 B}{k T}}}} .
\end{aligned}
$$

3. Die explizite Berechnung der absoluten Thermokraft

Je nach dem Ausdruck für die Fermi-Energie erhält man durch Einsetzen einer der Gln. (13), (13a), (13 b), (19), (19a), (19 b) in die allgemeine Beziehung (9) verschiedene Formeln für die absolute Thermokraft $\varepsilon$. Dabei ist die Gültigkeit der jeweiligen Nähe- 
rung für die Thermokraft auf das gleiche Temperaturintervall beschränkt wie die der entsprechenden Beziehung für $\zeta$. Quantitativ erhält man im reinen Störleitungsgebiet unter der Voraussetzung, daß $\zeta$ wenigstens $4 k T$ vom unteren Rand des Leitfähigkeitsbandes entfernt liegt, aus der vereinfachten Beziehung (9a):

$$
\begin{aligned}
\varepsilon=- & \frac{\Delta E_{\mathrm{D}}}{e T}-\frac{k}{e}\left\{\frac{1}{k T} \frac{I_{5 / 2}^{\mathrm{e}}}{I_{3 / 2}^{\mathrm{e}}}\right. \\
& -\ln \left(\frac{1}{2} \sqrt{\left.\left.1+4 \frac{n_{\mathrm{D}}}{n_{-}{ }^{0}} e^{\frac{\Delta E_{\mathrm{D}}}{k T}}-\frac{1}{2}\right)\right\} .}\right.
\end{aligned}
$$

Das ist die allgemeine Formel für die absolute Thermokraft eines einfachen Überschußhalbleiters in den Temperaturgebieten, in denen die Eigenhalbleitung noch nicht merklich ist.

Für die Näherung (13a) des $\zeta$-Wertes erhält man die bis auf das letzte Korrektionsglied bekannte Gleichung ${ }^{5}$ :

$$
\begin{aligned}
& \varepsilon=-\frac{\Delta E_{\mathrm{D}}}{2 e T}-\frac{k}{e}\left\{\frac{1}{k T} \frac{I_{5 / 2}^{\mathrm{e}}}{I_{3 / 2}^{\circ}}+\frac{1}{2} \ln \frac{n_{-}{ }^{0}}{n_{\mathrm{D}}}\right. \\
&\left.+\frac{1}{2}\left(\frac{n_{-}^{0}}{n_{\mathrm{D}}}\right)^{1 / 2} e^{-\frac{\Delta E_{\mathrm{D}}}{2 k T}}\right\} .
\end{aligned}
$$

Im Gebiet der vollständigen Ionisation folgt schließlich aus (13b) und (9a) für den reinen Überschußhalbleiter:

$\varepsilon=-\frac{k}{e}\left\{\frac{1}{k T} \frac{I_{5 / 2}^{\mathrm{e}}}{I_{3 / 2}^{\circ}}+\ln \frac{n_{-}{ }^{0}}{n_{\mathrm{D}}}+\frac{n_{\mathrm{D}}}{n_{-}{ }^{0}} e^{\frac{1 E_{\mathrm{D}}}{k T}}\right\}$.

Vollständig analog zu der Ableitung der Formeln für $\zeta$ gibt es auch für $\varepsilon$ eine Beziehung, die die absolute Thermokraft als Funktion der Elektronenkonzentration $n_{\mathrm{e}}$ im Leitungsband darstellt. Nur in dem durch Gl. (24a) erfaßten Gebiet darf dieses $n_{\mathrm{e}}$ in der üblichen Weise durch (17a) ersetzt werden. Aus (13c) und (9a) erhält man die in der Literatur vielfach benutzte Formel:

$$
\varepsilon=-\frac{k}{e}\left(\frac{1}{k T} \frac{I_{5 / 2}^{\mathrm{e}}}{I_{3 / 2}^{\mathrm{e}}}+\ln \frac{n_{-}^{0}}{n_{\mathrm{e}}}\right) .
$$

Diese Beziehung allein kann zur Bestimmung der scheinbaren Masse der Elektronen, die in $n_{-}{ }^{0}$ enthalten ist, im Zusammenhang mit dem Hall-Effekt benutzt werden. Doch ist die Verknüpfung der Hall-Konstanten mit der Ladungsträgerdichte in starkem Maße von der Art des Streumechanismus abhängig. So können nach Untersuchungen von Johnson und Lark-Horovitz ${ }^{6}$ die so bestimmten

5 R. H. Fowler, Statistical Mechanics, Cambridge Univ. Press, London 1936.
$n_{\mathrm{e}}$-Werte um den Faktor 2 differieren, je nachdem eine überwiegende Gitterstreuung oder Streuung an ionisierten Zentren vorliegt. Dadurch ist auch die Bestimmung des $m_{\mathrm{e}}{ }^{*}$ aus $(24 \mathrm{c})$ mit dieser Unsicherheit behaftet, es sei denn, man hat die Temperaturabhängigkeit der Beweglichkeit und so die Art des überwiegenden Streuprozesses ermittelt.

Demgegenüber sind die Beziehungen (24), (24a), (24b), abgesehen von dem meist sehr kleinen Anteil $(\mathrm{l} / k T)\left(I_{5 / 2}^{\mathrm{e}} / I_{3 / 2}^{\mathrm{e}}\right)$, von dieser Unsicherheit unabhängig. Hier gehen nur die experimentell leicht bestimmbaren Aktivierungsenergien und Donatorkonzentrationen ein, so daß die Ermittlung der scheinbaren Masse aus einer dieser Formeln einen etwas höheren Genauigkeitsgrad erhält.

Die angegebenen Gln. (24, bis (24c) können noch vereinfacht werden, wenn man Aussagen über den Streumechanismus machen kann. Im Grenzfall der rein thermischen Gitterstreuung wird $\tau_{\mathrm{e}}(E) \sim E^{-1 / 2}$; dann folgt durch einfache Rechnung:

$$
\frac{1}{k T} \frac{I_{5 / 2}^{\mathrm{e}}}{I_{3 / 2}^{\mathrm{e}}}=2 \text {. }
$$

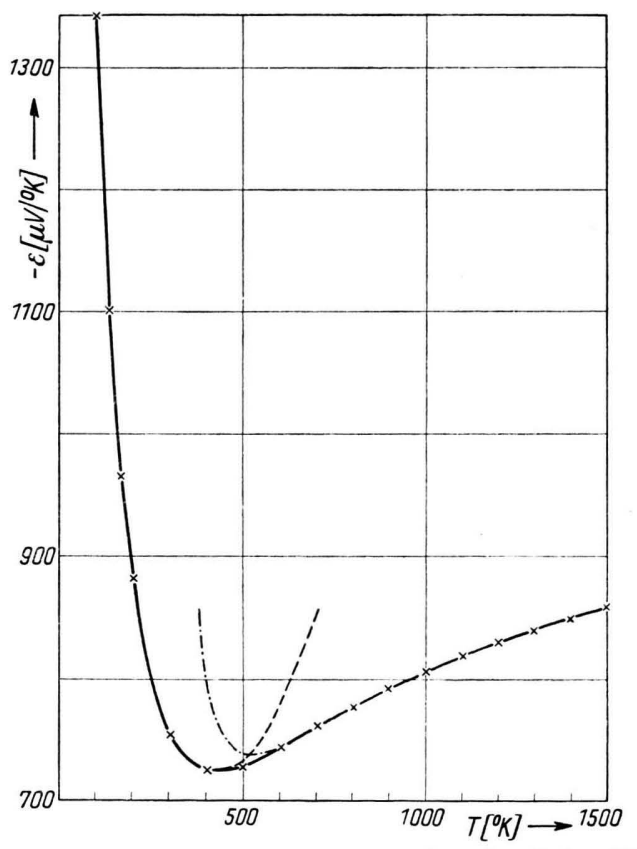

Abb. 3 a. Temperaturabhängigkeit der absoluten Thermokraft eines Überschußhalbleiters im Störleitungsgebiet. $\Delta E_{\mathrm{D}}=0,2 \mathrm{eV} ; n_{\mathrm{D}}=10^{17} \mathrm{~cm}^{-3}$. - - nach Gl. (24a), — - nach Gl. $(24 \mathrm{~b})$, — nach Gl. (24), $\times$ numerisch nach $(9 \mathrm{a}, 10 \mathrm{a})$ berechnete Werte.

${ }^{6}$ V. A. Johnson u. K. Lark-Horovitz, Physic. Rev. 79, 176 [1950]; H. Jones, Physic. Rev.\$1, 149 [1951]. 
In dem zweiten Grenzfall, der vornehmlich bei tiefen Temperaturen auftreten wird, werden die Elektronen an ionisierten Zentren gestreut, und es gilt ${ }^{7}$ $\tau_{\mathrm{e}}(E) \sim E^{3 / 2}$. Damit ergibt sich:

$$
\frac{1}{k T} \frac{I_{5 / 2}^{\mathrm{e}}}{I_{3 / 2}^{\mathrm{e}}}=4 \text {. }
$$

Unter der Voraussetzung rein thermischer Streuung ist in der Abb. 3 a der exakte Verlauf der Thermokraft eines Störstellenhalbleiters gemäß Gl. (24) festgelegt. Außerdem ist in der Abb. 3 b die Ab-

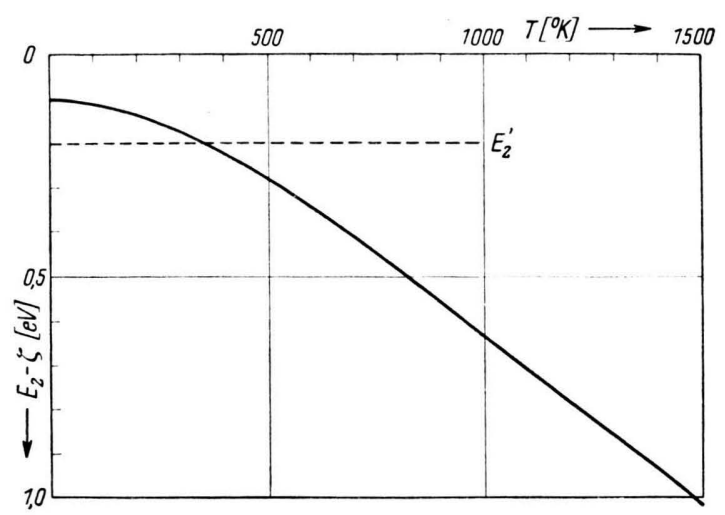

Abb. 3 b. Temperaturabhängigkeit der Fermischen Grenzenergie eines Überschußhalbleiters im Störleitungsgebiet. $\Delta E_{\mathrm{D}}=0,2 \mathrm{eV} ; n_{\mathrm{D}}=10^{17} \mathrm{~cm}^{-3}$.

nahme der Fermischen Grenzenergie als Funktion der Temperatur nach (13) dargestellt worden. Dabei sind $n_{\mathrm{D}}=10^{17} \mathrm{~cm}^{-3}$ und $\angle E_{\mathrm{D}}=0,2 \mathrm{eV}$. Die Eigenleitung soll in dem betrachteten Temperaturintervall so klein sein, daß sie keinen Einfluß auf die Thermokraft ausübt. Die gestrichelten Linien stellen die beiden Näherungslösungen (24a) und (24b) dar und geben so eine Anschauung von der Güte und dem Gültigkeitsbereich der benutzten Näherung. Außer der im allgemeinen erwarteten $A$ bnahmedes $\varepsilon$-Wertes mit wachsender Temperatur gibt es im Gebiet der vollständigen Ionisation einen Bereich, in dem $\varepsilon$ mit $T$ zunimmt. Die Größe dieses Intervalles hängt von der Störstellenkonzentration in dem Sinne ab, daß das Minimum von $\varepsilon$ nach um so höheren Temperaturen verschoben ist, je größer $n_{\mathrm{D}}$ wird. $\varepsilon_{\min }$ tritt ferner bei um so höherem $T$ auf, je größer $\Delta E_{\mathrm{D}}$ ist. Die obere Grenze für das Intervall, in dem $\varepsilon$ ansteigt, ist durch das Auftreten eines weiteren Störniveaus oder die beginnende Eigenhalbleitung bedingt. Beide Einflüsse können sich schon bei so tiefem $T$ bemerkbar machen, daß der ansteigende Ast vollständig unterdrückt wird.

Existiert ein weiteres Donatorenniveau mit einem größeren $\Delta E_{\mathrm{D}^{-}}$-Wert, so erhält man je nach der relativen Lage der Störniveaus zueinander nach dem ansteigenden Ast einen Abfall bis zu einem zweiten $\varepsilon_{\min }$ und von dort einen abermaligen Wiederanstieg, wenn auch in dem zweiten Niveau alle Donatoren ionisiert sind. Der Einfluß von Acceptortermen in der Nähe des Leitfähigkeitsbandes ist quantitativ schwieriger zu übersehen. Qualitativ können wir jedoch sagen, daß diese Terme im allgemeinen die Elektronenzahl herabsetzen werden und damit zu höheren $\varepsilon$-Werten Anlaß geben. Ist der Übergang der Elektronen in die Acceptorterme noch mit einer Aktivierungsenergie verknüpft, so wird der Abfall der $\varepsilon$-Werte mit wachsendem $T$ gegenüber dem Fall des reinen Überschußniveaus verzögert, der Wiederanstieg im Gebiet vollständiger Ionisation dagegen stark vergrößert.

Um nun den Anteil des Valenzbandes an der Thermokraft $\varepsilon$ mit zu erfassen, müssen wir auf die allgemeinere Gl. (9) zurückgreifen. Dabei wird der $\zeta$-Wert in dem ganzen in Betracht kommenden Gebiet durch die Beziehung (19) gegeben. Unter Beachtung von (11) folgt aus (9) mit (19):

$$
\begin{aligned}
& \varepsilon=-\frac{1}{e T} \\
& \frac{\left(m_{\mathrm{e}}{ }^{*}\right)^{1 / 2} \frac{n_{\mathrm{e}}}{n_{-}{ }^{0}}\left[I_{5 / 2}^{\mathrm{e}}-k T \ln \frac{n_{\mathrm{D}}}{n_{-}{ }^{0}}-k T \ln (1+p)\right]-\left|m_{\mathrm{h}}{ }^{*}\right|^{1 / 2} \frac{n_{\mathrm{h}}}{n_{+}{ }^{0}}\left[I_{5 / 2}^{\mathrm{h}}+\Delta B+k T \ln \frac{n_{1}}{n_{-}{ }^{0}}+k T \ln (1+p)\right]}{\left(m_{\mathrm{e}}{ }^{*}\right)^{1 / 2} \frac{n_{\mathrm{e}}}{n_{-}{ }^{0}} I_{3 / 2}^{\mathrm{e}}+\left|m^{*}{ }_{\mathrm{h}}\right|^{1 / 2} \frac{n_{\mathrm{h}}}{n_{+}{ }^{0}} I_{3 / 2}^{\mathrm{h}}},
\end{aligned}
$$

wobei zur Kürzung

gesetzt sei.

$$
p=-\frac{1}{2}+\frac{1}{2} \sqrt{1+4 \frac{n_{+}{ }^{0} n_{-}{ }^{0}}{n_{\mathrm{D}}{ }^{2}} e^{-\frac{A B}{k T}}}
$$

${ }^{7}$ E. Conwell u. V. F. Weißkopf, Physic. Rev. 77, 388 [1950]. 
Zur Vereinfachung dieser Beziehung wollen wir berücksichtigen, daß der Nenner von (25) bis auf einen konstanten Faktor gleich der elektrischen Leitfähigkeit des betrachteten Halbleiters ist. Diese können wir aber durch

$$
\sigma=e\left(n_{\mathrm{e}} \mu_{\mathrm{e}}+n_{\mathrm{h}} \mu_{\mathrm{h}}\right)
$$

darstellen, wenn $\mu_{\mathrm{e}}, \mu_{\mathrm{h}}$ die Beweglichkeiten der Ladungsträger bedeuten. Diese werden

$\mu_{\mathrm{e}}=C \cdot \frac{\left(m_{\mathrm{e}}{ }^{*}\right)^{1 / 2}}{n_{-}{ }^{0}} I_{3 / 2}^{\mathrm{e}}, \mu_{\mathrm{h}}=C \cdot \frac{\left|m_{\mathrm{h}}{ }^{*}\right|^{1 / 2}}{n_{+}{ }^{0}} I_{3 / 2}^{\mathrm{h}}$.

Als Abkürzung sei der Quotient

$$
q=\frac{\mu_{\mathrm{e}}}{\mu_{\mathrm{h}}}=\frac{\left(m_{\mathrm{e}}{ }^{*}\right)^{1 / 2}}{\left|m_{\mathrm{h}}{ }^{*}\right|^{1 / 2}} \frac{n_{+}{ }^{0}}{n_{-}{ }^{0}} \frac{I_{3 / 2}^{\mathrm{e}}}{I_{3 / 2}^{\mathrm{h}}}
$$

eingeführt. Dann folgt aus (25) unter Berücksichtigung von (23) und (26):

$\varepsilon=-\frac{1}{e T} \frac{(1+p) q\left(I_{5 / 2}^{\mathrm{e}} / I_{3 / 2}^{\mathrm{e}}\right)-p\left(I_{5 / 2}^{\mathrm{h}} / I_{3 / 2}^{\mathrm{h}}\right)}{(1+p) q+p}$

$+\frac{\Delta B}{e T} \frac{p}{q(1+p)+p}+\frac{k}{e} \ln \frac{n_{\mathrm{D}}}{n_{-}{ }^{0}}+\frac{k}{e} \ln (1+p)$.

Im Eigenleitungsgebiet wird in den meisten Fällen die thermische Gitterstreuung ausschlaggebend sein, so daß mit $\tau \sim E^{-1 / 2}$ für Elektronen und Defektelektronen

$$
I_{5 / 2}^{\mathrm{e}} / I_{3 / 2}^{\mathrm{e}}=I_{5 / 2}^{\mathrm{h}} / I_{3 / 2}^{\mathrm{h}}=2 k T
$$

ist. Also folgt:

$$
\begin{aligned}
\varepsilon=-\frac{k}{e}\left\{2-\ln \frac{n_{\mathrm{D}}}{n_{-}{ }^{0}}\right. & -\left(4+\frac{\Delta B}{k T}\right) \frac{p}{q(1+p)+p} \\
- & \ln (1+p)\}
\end{aligned}
$$

als allgemeine Formel für die absolute Thermokraft eines Halbleiters im Störstellen- und Eigenleitungsgebiet, sofern nur $\zeta$ wenigstens $4 k T$ unter dem Donatorniveau liegt.

Für die Grenzfälle (19a) und (19b) bei der Berechnung der Fermi-Energie erhält man aus (31)

$$
\begin{array}{r}
\varepsilon=-\frac{k}{e}\left\{2-\ln \frac{n_{\mathrm{D}}}{n_{-}{ }^{0}}-\left(\frac{n_{-}{ }^{0}}{n_{\mathrm{D}}}\right)^{2} e^{-\frac{\Delta B}{k T}}\left(\frac{\left|m_{\mathrm{h}}{ }^{*}\right|}{m_{\mathrm{e}}{ }^{*}}\right)^{1 / 2}\right. \\
\left.\cdot\left(4+\frac{\Delta B}{k T}+\frac{\left|m_{\mathrm{h}}{ }^{*}\right|}{m_{\mathrm{e}}{ }^{*}}\right)\right\} \quad \text { (31 a) }
\end{array}
$$

unter der Bedingung, daß sich der Überschußleitung eine geringe Eigenleitung überlagert, und

$$
\begin{aligned}
& \varepsilon=-\frac{k}{e}\left\{\frac{\Delta B}{2 k T} \frac{\bar{q}-1}{\bar{q}+1}+2 \frac{\bar{q}-1}{\bar{q}+1}\right. \\
& \left.-\frac{3}{4} \ln \left(\frac{\left|m_{\mathrm{h}}{ }^{*}\right|}{m_{\mathrm{e}}{ }^{*}}\right)-\frac{1}{2} \frac{n_{\mathrm{D}}}{\left(n_{-}{ }^{0} n_{+}{ }^{0}\right)^{1 / 2}} e^{\Delta B / 2 k T}\right\},
\end{aligned}
$$

sofern zu der Eigenhalbleitung noch Elektronen aus Donatortermen hinzutreten. Dabei ist zur Abkürzung

$$
\bar{q}=q \frac{p+1}{p}
$$

eingeführt worden. Bemerkenswert ist an der Gl. (31 b) die bis auf den letzten Summanden vollständige Übereinstimmung mit der entsprechenden Beziehung für den idealen Eigenhalbleiter, sofern man nur $\bar{q}$ durch $q$ ersetzt. Für letzteren gilt:

$$
\begin{aligned}
& \varepsilon=-\frac{k}{e}\left\{\frac{\Delta B}{2 k T} \frac{q-1}{q+1}\right.+2 \frac{q-1}{q+1} \\
&\left.-\frac{3}{4} \ln \left(\frac{\left|m_{\mathrm{h}}{ }^{*}\right|}{m_{\mathrm{e}}{ }^{*}}\right)\right\} .
\end{aligned}
$$

Da der erste Summand in der Klammer von (31 b) in den meisten Fällen den größten Wert hat, können wir in erster Näherung den Einfluß von Störstellen formal durch eine Änderung der scheinbaren Massen beschreiben. Dabei ergibt sich für das Verhältnis dieser Massen eine temperaturabhängige Größe $\bar{q}$, die mit wachsendem $T$ asymptotisch den Wert $\left|m_{\mathrm{h}}{ }^{*}\right| / m_{\mathrm{e}}{ }^{*}$ erreicht. Quantitativ gilt in der Näherung $(31 \mathrm{~b})$

$$
p \approx \frac{\left(n_{+}{ }^{0} n_{-}{ }^{0}\right)^{1 / 2}}{n_{\mathrm{l}}} e^{-\frac{\Delta B}{2 k T}}
$$

und damit

$$
\bar{q}=\frac{\left|m_{\mathrm{h}}{ }^{*}\right|}{m_{\mathrm{e}}{ }^{*}}\left(1+\frac{n_{\mathrm{D}}}{\left(n_{-}{ }^{0} n_{+^{0}}\right)^{1 / 2}} e^{\frac{\Delta B}{2 k T}}\right) .
$$

Das Vorhandensein der Überschußelektronen aus dem Donatorterm wirkt sich also so aus, als ob die scheinbare Masse der Elektronen im Vergleich zu der der Defektelektronen verkleinert wäre, wodurch die Beweglichkeiten im umgekehrten Verhältnis bei den Elektronen gegenüber den Defektelektronen vergrößert erscheint.

In der Abb. 4 ist die Temperaturabhängigkeit der absoluten Thermokraft eines Halbleiters dargestellt, dessen $\zeta$-Werte bereits in der Abb. 2 als Funktion der Temperatur aufgetragen sind. Das berechnete Beispiel entspricht etwa dem einer Ge-Probe. Dabei sind die gemäß Gl. (9) exakt berechneten $\varepsilon$-Werte als Punkte eingetragen. Die ausgezogenen Kurven sind gemäß Gl. (31) bzw. im Störleitungsgebiet gemäß Gl. (24) berechnet worden. Außerdem sind gestrichelt die Näherungslösungen (31 a) bis (31 c) eingetragen, die für sich schon eine recht gute Berechnung der Thermokraft im Übergangsgebiet ermöglichen. Wo sich mehrere Kurven überdecken, ist in 
der Abb. 4 der Übersichtlichkeit halber nur die nach (31) bzw. (24) berechnete eingezeichnet worden.

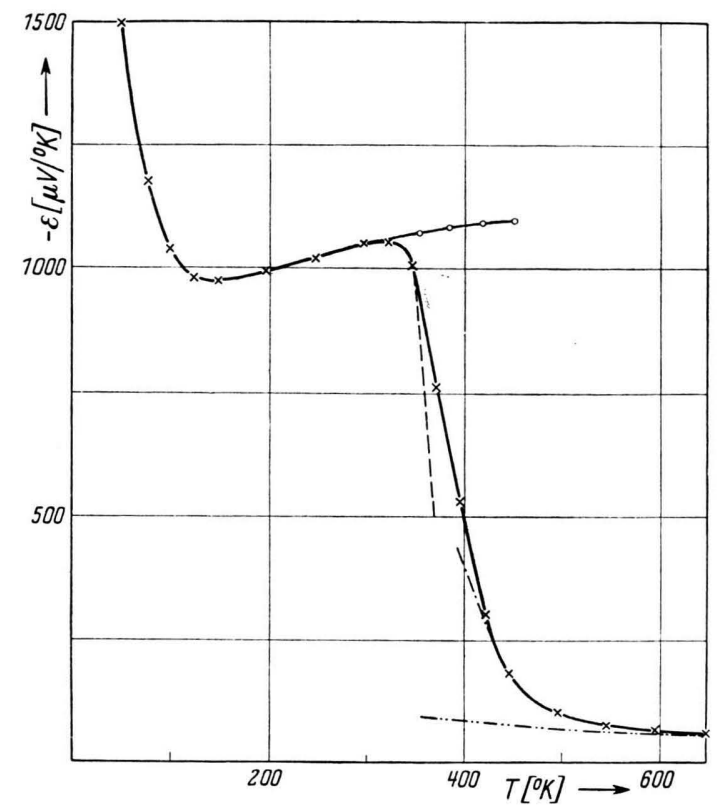

Abb. 4. Temperaturabhängigkeit der absoluten Thermokraft eines Überschußhalbleiters. $\left|m_{\mathrm{h}}^{*}\right| / m_{\mathrm{e}}^{*}=1,2$; $n_{\mathrm{\nu}}=10^{15} \mathrm{~cm}^{-3} ; \Delta B=0,74 \mathrm{eV} ; \Delta E_{\mathrm{D}}=0,1 \mathrm{eV} . \overline{\mathrm{Cl}}$ nach Gl. (24) bzw. (31), — — nach Gl. (31a), —— nach Gl. (31 b), _ - - nach Gl. (31 c), - — nach Gl. $(24), \times$ numerisch nach $(9,10 \mathrm{a})$ berechnete Werte.

Das gut ausgeprägte Maximum in der Abb. 4 kann bei bekanntem $\Delta B$ zur Berechnung des Verhältnisses der scheinbaren Massen der Ladungsträger benutzt werden. Durch Differentiation von (31) und Nullsetzen der ersten Ableitung erhält man eine algebraische Gleichung für $q$, die numerisch zu lösen ist. Diese Berechnung des $q$-Wertes hat den großen Vorteil für sich, daß man die Messungen nicht bis in das oft schwer zugängliche Eigenleitungsgebiet auszudehnen braucht.

Abschließend wollen wir noch einen Sonderfall diskutieren. So erhält man in vollständig analoger Rechnung für einen Eigenhalbleiter, bei dem durch einen Acceptorterm in der Nähe des Valenzbandes eine Defektleitung überlagert ist, in einer Gl. (31 c) entsprechenden Näherung

$$
\begin{aligned}
\varepsilon & =-\frac{k}{e}\left\{\frac{\Delta B}{2 k T} \frac{\overline{\bar{q}}-1}{\overline{\bar{q}}+1}+2 \frac{\overline{\bar{q}}-1}{\overline{\bar{q}}+1}\right. \\
& \left.-\frac{3}{4} \ln \left(\frac{\left|m_{\mathrm{h}}{ }^{*}\right|}{m_{\mathrm{e}}{ }^{*}}\right)+\frac{1}{2} \frac{n_{\mathrm{A}}}{\left(n_{-}{ }^{0} n_{+}{ }^{0}\right)^{1 / 2}} e^{\frac{\Delta B}{2 k T}}\right\}
\end{aligned}
$$

mit

$$
\overline{\bar{q}}=\frac{\left|m_{\mathrm{h}}{ }^{*}\right|}{m_{\mathrm{e}}{ }^{*}}\left(1-\frac{n_{\mathrm{A}}}{\left(n_{-}{ }^{0} n_{+}{ }^{0}\right)^{1 / 2}} e^{\frac{\Delta B}{2 k T}}\right) .
$$

Dabei ist insbesondere der Fall interessant, daß $\left|m_{\mathrm{h}}{ }^{*}\right|=m_{\mathrm{e}}{ }^{*}$ wird. Dann gilt nämlich:

$$
\begin{aligned}
\varepsilon=\frac{k}{e}\left\{\frac{\Delta B}{2 k T}\right. & \frac{n_{\mathrm{A}}}{2\left(n_{-}{ }^{0} n_{+}{ }^{0}\right)^{1 / 2}} e^{\frac{\Delta B}{2 k T}} \\
+ & \left.\frac{n_{\mathrm{A}}}{2\left(n_{-}{ }^{0} n_{+}{ }^{0}\right)^{1 / 2}} e^{\frac{\Delta B}{2 k T}}\right\}>0
\end{aligned}
$$

für alle $T$. Also kann $\varepsilon$ theoretisch auch im Eigenleitungsgebiet positiv bleiben und braucht nicht beim Übergang von der Störstellen- zur Eigenhalbleitung das Vorzeichen zu wechseln. Doch nimmt in diesen einfachen Modellen bei genügend hohen Temperaturen $|\varepsilon|$ stets ab.

Die vorstehend mitgeteilte Methode zur Bestimmung des $\zeta$-Wertes im Übergangsgebiet von der Störstellen- zur Eigenhalbleitung kann noch etwas verallgemeinert werden. So kann man die Rechnungen auf den Fall ausdehnen, daß der Übergang zur Eigenhalbleitung schon erfolgt, wenn man sich bezüglich der Störstellen noch nicht im Gebiet der vollständigen Ionisation befindet, sondern erst nahezu 50\% der Störstellen ihre Elektronen abgespalten oder aufgenommen haben. Dann werden die $\zeta$-Werte und damit die Thermokraftformeln auch im Übergangsgebiet von der Aktivierungsenergie $\Delta E_{\mathrm{D}}$ abhängig. Doch bringen diese Rechnungen keine wesentliche Erweiterung der vorliegenden Darstellung, so daß auf eine Wiedergabe verzichtet werden soll.

Die hier mitgeteilten Rechnungen werden in einigen einfachen Fällen ausreichen, um die experimentellen Ergebnisse hinreichend genau zu beschreiben. Nur muß man sich in jedem einzelnen Fall davon überzeugen, ob wirklich nur eine Aktivierungsenergie auftritt und die Voraussetzungen $\Delta E_{\mathrm{D}} \leq 2 / 3$ $(\Delta B / 2)$ bzw. $E_{2}-\zeta \geq 4 k T$ bzw. $\zeta-E_{1} \geq 4 k T$ auch erfüllt sind.

4. Vergleich mit einigen experimentellen Ergebnissen

Ein genauer Vergleich zwischen Theorie und Experiment wird gerade bei der Thermokraft durch den Einfluß von Oberflächenschichten, inhomogen verteilten Störstellenkonzentrationen, inneren Sperrschichten u. a. sehr erschwert. Auch fehlen häufig bei den in der Literatur veröffentlichten Meßergebnissen z. B. die Angaben über die Donator- und Acceptorkonzentrationen, die einen erheblichen Ein- 
fluß auf die Lage des Fermi-Niveaus und damit auf die Gültigkeitsgrenzen einer der hier mitgeteilten Thermokraftformeln ausüben.

Im Gebiet der Störstellenhalbleitung liegen zahlreiche Meßresultate von den verschiedensten Stoffen vor. Doch müssen wir zu einem Vergleich mit den obigen Formeln alle die Substanzen ausscheiden, bei denen die Aktivierungsenergie in dem betrachteten Temperaturintervall kleiner als $8 k T$ ist. Für diese Substanzen dürften die bereits zitierten Überlegungen von $\mathrm{Yamashita}{ }^{2}$ besser zutreffen. Außerdem bleiben alle die Messungen für unsere Betrachtungen uninteressant, bei denen ein ausgeprägtes Störstellenniveau mit relativ hoher Störstellenkonzentration in bezug auf die Thermokraft nur den ersten abfallenden Ast der Abb. 3 hervortreten läßt. Für unsere theoretischen Überlegungen sind die Meßergebnisse an Halbleitern mit mehreren Störstellenniveaus und einer überlagerten Eigenhalbleitung bedeutsamer. So sei z. B. auf die Experimente von Morin ${ }^{8}$ an reinem und mit $\mathrm{Ti}$ versetztem $\alpha \mathrm{Fe}_{2} \mathrm{O}_{3}$ verwiesen. Aus dem elektrischen Verhalten einzelner Proben müssen wir schließen, daß bei diesen die Voraussetzung einer Nichtentartung des Elektronengases erfüllt ist. Gerade diese Proben zeigen aber die in der Abb. 4 dargestellte Temperaturabhängigkeit. Der starke Abfall nach einem leicht ansteigenden Kurventeil ist in dem Diagramm der zitierten Arbeit deutlich sichtbar. Leider können diese Messungen wohl nicht bis zu so hohen Temperaturen fortgesetzt werden, daß man auch bei den mit Ti versetzten Proben bezüglich der Thermokraft den Übergang zur Eigenhalbleitung hin quantitativ verfolgen kann. Die schwache $\mathrm{Zu}$ nahme der Thermokraft einzelner Proben vor dem diskutierten Abfall entspricht auch quantitativ der vorliegenden Theorie.

Nachdem von Justi und Lautz gezeigt worden ist $^{9}$, daß auch intermetallische Verbindungen wie z. B. CdSb, echte Halbleiter sein können, dürfen

${ }^{8}$ F. J. Morin, Physic. Rev. 83, 1005 [1951].

${ }^{9}$ E. Justi u. G. Lautz, Z. Naturforschg. 7 a, 191 u. 602 [1952]. wir für unseren Vergleich zwischen den angegebenen Thermokraftformeln und einigen bekannten Meßergebnissen auch die Temperaturabhängigkeit der $\varepsilon$-Werte von CdSb mit einbeziehen. So zeigen schon die Kurven von Fischer und Pfleiderer ${ }^{10}$ bei Zimmertemperatur zunächst einen schwachen Anstieg und zu höheren Temperaturen hin einen starken Abfall. Doch ist die Streubreite der Einzelmessungen für eine quantitative Auswertung zu groß.

Schließlich möchten wir noch auf ausgedehnte $\varepsilon$ Messungen von $\mathrm{Henkels}{ }^{11}$ am Se eingehen, bei dem bei Zimmertemperatur ein Anstieg der Thermokraft mit zunehmendem $T$ beobachtet wird. Erst im flüssigen Zustand wird ein Maximum durchlaufen, dem eine Abnahme von $\varepsilon$ mit weiter steigendem $T$ folgt. Ähnlich wie bei den anderen elektrischen Eigenschaften des Se ergibt sich auch bei der Thermokraft keine quantitative Übereinstimmung mit der bestehenden Halbleitertheorie. So fällt der Anstieg von $\varepsilon$ mit $T$ gegenüber unseren Formeln um den Faktor $6-8$ zu groß aus. Hier könnten Elektronenspenderstellen in der Nähe des Valenzbandes und der Acceptorterme für einen solchen Effekt verantwortlich sein, wie im vorhergehenden Abschnitt diskutiert wurde.

Abschließend dürfen wir also feststellen, daß der qualitative Charakter des Verlaufs der Thermokraft als Funktion der Temperatur in vielen Fällen mit den theoretischen Formeln im Einklang steht. Zur quantitativen Auswertung bedarf es jedoch noch einer weiteren Kenntnis anderer Halbleiterkonstanten, so z. B. des Bandabstandes, der Aktivierungsenergien, der Donatorenkonzentrationen usf., wobei jeweils eine oder zwei dieser Größen den Thermokraftmessungen entnommen werden können, sofern die übrigen schon bekannt oder doch näherungsweise bekannt sind.

Abschließend möchte ich Herrn Professor Dr. M. Kohler für zahlreiche anregende Diskussionen und die Durchsicht dieses Manuskriptes herzlichst danken.

10 F. Fischer u. G. Pfleiderer, Gesammelte Abh. Kenntn. Kohle IV, 440 [1919].

${ }^{11}$ H. W. Henkels, Physic. Rev. 77, 735 [1950]. 